\title{
Trastuzumab/Pertuzumab/Vinorelbine Regimen
}

National Cancer Institute

\section{Source}

National Cancer Institute. Trastuzumab/Pertuzumab/Vinorelbine Regimen. NCI

Thesaurus. Code C156380.

A regimen consisting of trastuzumab, pertuzumab, and vinorelbine that may be used as a treatment for recurrent and metastatic HER-2 (EGFR2; ErbB2)-positive advanced or metastatic breast cancer. 